\title{
El factor humano y las relaciones laborales en las estrategias organizativas de downsizing
}

\author{
M. ELENA ROBLES RABAGO * \\ M. LUCÍA RODRÍGUEz FÉLXX *
}

\begin{abstract}
En la última década el desempleo se ha convertido en uno de los principales problemas de la sociedad española, con una especial incidencia sobre la fuerza de trabajo andaluza. De todos los fenómenos que, directa o indirectamente, han derivado en despidos masivos de personal, quizás sea el Downsizing el más polémico y desconocido. Es por este motivo que el objetivo de este artículo haya sido el definir y acotar el significado de este término para recoger a continuación los efectos que su implantación ha provocado tanto en la organización como en el personal que, de una u otra forma, se ha visto afectado por el mismo. Hemos querido finalizar mostrando algunas de las alternativas que ciertas empresas han empleado en un esfuerzo por minimizar, en lo posible, las consecuencias negativas de esta estrategia sobre su fuerza laboral.
\end{abstract}

\section{Introducción}

El desempleo se ha convertido en uno de los principales problemas que ha atraído la atención de la sociedad española de manera especial en los últimos tiempos.

El despido no es un fenómeno abstracto que ocurra sólo en ciertas empresas, negocios, profesiones o sectores de actividad, es un fenómeno generalizado. Problemas de desindustrialización en ciertas regiones, reconversión en ciertos sectores (astilleros, altos hornos, etc...), fusiones y adquisiciones de

* Dpto. Adm. Empresas y Marketing. Escuela de Relaciones Laborales. Universidad de Sevilla. 
empresas tanto en el sector productivo como en el sector servicios unido a movimientos de reducción del tamaño (downsizing) en algunas empresas de mediano y gran tamaño, con la consiguiente eliminación de posiciones directivas y de trabajos administrativos (cuello blanco), han contribuido a una. pérdida de empleo a gran escala.

De estos fenómenos, el downsizing es el que más polémica ha suscitado entre los investigadores al asociarse de manera generalizada con los despidos. Es cierto que en muchas ocasiones el downsizing conlleva reducciones de personal pero ello no implica necesariamente una política exclusiva de despidos. Por ello, y dada la vigencia que en los últimos años ha cobrado este fenómeno, se hace imprescindible la definición y delimitación del significado e implicaciones de esta estrategia.

\section{Concepto de Downsizing}

El Downsizing organizacional comprende un conjunto de actividades que son emprendidas por parte de la dirección y que están encaminadas a la mejora de la eficiencia organizacional, de la productividad y/o de la competitividad. Representa una estrategia que afecta al tamaño de la fuerza de trabajo de la firma y a sus procesos de trabajo.

Superficialmente, el Downsizing puede ser interpretado simplemente como una reducción del tamaño organizacional. Cuando esto ocurre, el Downsizing se confunde a menudo con el concepto de declive, el cual, analizado también ligeramente puede interpretarse como una mesa reducción en el tamaño de la organización. Sin embargo, existen importantes diferencias que hacen al Downsizing y al declive fenómenos distintos conceptual y empíricamente. Las organizaciones pueden entrar en una situación de declive cuando fracasan en la anticipación, reconocimiento, prevención, neutralización, o adaptación a las presiones externas o internas que amenazan su supervivencia a largo plazo. En este sentido, el Downsizing se convierte en una estrategia a implantar para evitar precisamente el declive mediante la adopción de las medidas adecuadas.

Por ello, el Downsizing se ha identificado comúnmente con procesos cuyo . objeto consiste en reducir, comprimir o consolidar la organizacion, todo 10 cual se ha venido considerando a su vez como un último esfuerzo para evitar la desaparición de la misma o para ajustarse temporalmente a una caída cíclica de las ventas.

La estrategia de Downsizing ha tenido generalmente conotaciones negativas pues ha sido dirigida casi siempre al despido de los empleados «cuello 
azul" o a empleados contratados por horas, convirtiendo a este fenómeno en algo no deseado (véase Hirschorn and associates. 1983 y la revista Focus, vol. $71,1994)$. Sin embargo, las recesiones de principio de los 80 y 90 , unidas al largo deterioro en la competitividad global de las empresas americanas y europeas, han provocado una debilidad potencial en ellas; muchas firmas están sobrecargadas de personal, son pesadas, lentas e ineficientes, de ahí que los directivos se hayan replanteado las vías de organizar y gestionar sus compañías. El Downsizing se ha convertido, por consiguiente, en una más de las estrategias a elegir, sobre todo, en las grandes organizaciones.

\section{Atributos clave del Downsizing}

$1^{\circ}$. El Downsizing no es algo que surja de forma espontánea en las organizaciones sino que es algo que éstas emprenden deliberadamente, siendo por tanto un conjunto intencionado de actividades, lo cual lo diferencia de otros acontecimientos como: pérdidas de cuota de mercado, pérdidas en ingresos o pérdidas involuntarias de recursos humanos, procesos éstos que van asociados con el declive organizacional. El Downsizing, por tanto, implica una acción organizacional en muchos casos previsional.

$\mathbf{2}^{\circ}$.El Downsinzing conlleva corrientemente "reducciones de personal", no obstante no se limita exclusivamente a esta estrategia. Aunque existe una variedad de estrategias de reducción de personal que sí están asociadas con el Downsizing, tales como: transferencias, recolocación externa (outplacement), bajas incentivadas, indemnizaciones, despidos, etc..., el Downsizing no siempre supone reducciones de personal. No obstante, dado que existen situaciones en las cuales se incorporan nuevos productos, nuevos recursos o fuentes de ingreso, o se asumen tareas adicionales sin incrementar ostensiblemente el número de empleados, nos encontramos con la necesidad de menos trabajadores por unidad producida en relación con situaciones precedentes.

$3^{\circ}$.El Downsizing se centra en la mejora de la "eficiencia" de la organización y puede emprenderse de forma proactiva o reactiva con el objeto de contener los costes, incrementar los ingresos o para reforzar la competitividad. Es decir, puede ser puesto en práctica como una reacción defensiva ante el declive o como una estrategia proactiva para incrementar el rendimiento organizacional. En cualquier caso, el Downsizing se diseña normalmente para contener los costes o para disminuirlos. 
$4^{\circ}$. El Downsizing afecta, consciente o inconscientemente, a los «procesos de trabajon. Cuando la fuerza de trabajo se reduce, por ejemplo, si dejamos un menor número de empleados para que realicen la misma cantidad de trabajo, esto puede tener un impacto sobre qué trabajo debe hacerse y cómo debe hacerse.

\section{Diferenciación entre el Downsizing y otros conceptos colaterales}

Algunos atributos importantes del Downsizing también lo diferencian de otros conceptos relacionados tales como: el crecimiento a la inversa (growth-in-reverse), la inadaptación (nonadaptation) o el despido (layoffs).

\section{Distinción entre Downsizing y Growth-in-reverse (crecimiento a la inversa)}

Debido a que lo contrario al Downsizing es el crecimiento, algunos autores han supuesto que el Downsizing es sinónimo del término opuesto al crecimiento, lo que implica asociarlo con la dinámica inversa implícita en el proceso de expansión organizacional.

Cuando una organización crece, por ejemplo, se han identificado una serie de patrones predecibles de etapas que suponen un conjunto de resultados organizacionales tales como: descentralización, especialización e incremento del número de unidades que amplían sus límites (véase Blau 1970, Greines 1972, Quinn y Cameron 1983). Por ello, algunos autores han sugerido que el Downsizing implica la dinámica contraria a la anterior, o sea, una secuencia opuesta a las etapas de crecimiento y lo contrario a los procesos de crecimiento, esto es: más centralización, menos especialización y menos ampliación de los límites (véase Behn, 1980, Gilmore y Hirschorn 1983, Krantz 1985). Sin embargo, ni estas etapas ni estos resultados organizacionales están asociados necesariamente con el Downsizing.

La naturaleza intencional del Downsizing significa que una organización puede hacerse más pequeña para descentralizar, especializarse o llegar a estar más relacionada con el exterior a través de actividades de ampliación de sus límites, es decir, el Downsizing conlleva los mismos resultados que están asociados con el crecimiento. Así, por ejemplo, el downsizing puede efectuarse recortando las funciones corporativas para conseguir una mayor descentralización, estableciendo unidades especiales para atender a diversos 
lugares corporativos (especialización), o involucrando a organizaciones externas en la planificación e implantación del Downsizing, todo ello encaminado fundamentalmente, a extender más los límites corporativos.

En resumen, el Downsizing y el crecimiento pueden, teóricamente por lo menos, crear las mismas formas y estructuras organizacionales y un Downsizing efectivo puede conducir al crecimiento.

\section{Distinción entre Downsizing y Nonadaptation (Inadaptación)}

Siguiendo con las diferencias entre el Downsizing y otros términos con los cuales se le confunde habitualmente, algunos autores han definido también al Downsizing como lo contrario a la adaptación y han situado la inadaptación como un concepto clave a la hora de definirlo (véase Gilmore y Hirschorn 1983, McKinley 1987, Weitzel y Johnsson 1989).

El Downsizing, per se, no implica ni mala adaptación, ni fallos, ni un bajo nivel de ejecución, sino que representa un movimiento estratégico en una parte de la organización para incrementar su ejecución en relación al entorno en el que se está desenvolviendo. Este movimiento estratégico puede ser proactivo y reactivo (distinción hecha originalmente por Miles y Cameron, 1982). Pero, incluso en el Downsizing reactivo, no están implicadas ni la ineficiencia ni la inadaptación. El Downsizing puede ser una reacción ante ciertos pasos erróneos o ante limitaciones ambientales o, en los casos proactivos, puede ser una acción previa para mejorar la ejecución organizacional.

\section{Distinción entre Downsizing y Layoffs (Despidos)}

Una manifestación común del Downsizing es el despido de empleados o la expulsión de trabajadores, con o sin previo aviso. Debido a que los despidos han sido tradicionalmente (y desafortunadamente siguen siendo) la primera alternativa empleada para el Downsizing, algunos autores han tratado el despido y el Downsizing como fenómenos sinónimos (Véase Gilmore y Hirschorn 1983, Brockner 1988).

En este sentido, McCune, Beatty y Montagno (1988) encontraron, en una investigación realizada sobre cien empresas manufactureras del medio oeste norteamericano, que se habían tenido en cuenta pocas alternativas diferentes al despido en cualquiera de esta firmas y que el $94 \%$ de los directivos planificaron y pusieron en práctica los despidos en un período inferior a 60 días. Por tanto, el despido fue la opción seleccionada abrumadoramente frente a la falta de otras alternativas para el Downsizing. 
Sin embargo, el Downsizing se diferencia del despido por ser un concepto más amplio. Así, mientras el despido hace referencia a una táctica simple (operación reactiva utilizada para poner en práctica una estrategia de Downsizing), el Downsizing puede ser tanto una operación reactiva como proactiva. El Downsizing incluye una serie de opciones para reducir la fuerza de trabajo además del despido, de hecho, el despido puede no incluirse en absoluto en una estrategia de Downsizing organizacional.

Las investigaciones sobre el Downsizing se centran en reducciones a partir de un nivel de análisis organizacional, mientras aquéllas que se hacen sobre el despido se centran en un nivel de análisis individual (véase Brockner 1988).

En conclusión, el Downsizing es un concepto que debería tratarse de forma separada de otros conceptos asociados con la dinámica del declive, tales como ineficiencia, despidos o simple contracción de la organización.

V. ¿Es posible una implantación adecuada del Downsizing?

La razón que subyace tras los despidos de los últimos años parece ser la reduccion del personal, comenzando por las capas de mayor edad. Para ello, el método directo más utilizado por el Downsizing ha sido la eliminación de empleados a través de jubilaciones anticipadas, colocación externa, traslado a otras unidades, reemplazo de empleados regulares con contratos de ayuda temporal y, por último, despidos. Se buscaba de este modo un incremento de los beneficios económicos y organizacionales, sin embargo, normalmente el Downsizing se ha acometido imprudentemente y no se han conseguido estos beneficios (Margulis, 1994).

Para que el Downsizing consiga los beneficios esperados se deben identificar las razones reales de los problemas y sólo entonces diseñar e implantar la solución estratégica apropiada.

Según Buhler (1994), el Downsizing necesitaría comenzar con una cuidadosa planificación y visión de futuro. Dicho plan debería comunicarse adecuadamente a todos los departamentos y divisiones afectadas, o a toda la organización. Este primer paso es el que va a condicionar probablemente el éxito del Downsizing. Un segundo paso, y decisivo también, se centraría en el papel desempeñado por la dirección, la cual debería mantener la moral y la satisfacción de los empleados supervivientes.

Señala el autor que el mayor error cometido en la implantación del Downsizing es el de las reducciones de tamaño indiscriminadas a lo largo de toda la organización pues, como se ha podido comprobar, esta estrategia no da resultado en la mayoría de los casos. Asimismo debería ponerse un gran 
cuidado en la evaluación de los costes, de los puestos de trabajo vitales y de las funciones principales de la empresa antes de iniciar el recorte.

En relación a lo anterior cabe señalar la existencia de algunas investigaciones que han puesto de manifiesto los errores más frecuentes cometidos por las organizaciones al emprender procesos de Downsizing junto a otras investigaciones que sugieren diversas tácticas positivas de implantación.

Así, tras un estudio llevado a cabo por Moravec en 1994 sobre empresas que han acometido procesos de Downsizing, se identificaron algunos errores muy frecuentes cometidos por las mismas:

1. No comunicar al personal una visión clara de los objetivos específicos que se pretenden alcanzar.

2. Ignorar otras alternativas al Downsizing que, normalmente, pueden existir.

3. Torpe manejo de los factores emocionales del personal.

4. Ignorar los efectos que el Downsizing puede tener sobre otros agentes sociales tales como: clientes, proveedores, socios, sindicatos, etc...; y consecuentemente, sobre la imagen de la empresa.

5. No atender debidamente a los trabajadores despedidos o desplazados por el Downsizing.

En este sentido, y basándose en un amplio trabajo de campo mediante entrevistas, Michael Hitt y asociados (1994) identificaron algunas tácticas de Downsizing tanto eficaces como ineficaces. Entre las ineficaces encontraron las bajas voluntarias incentivadas y los despidos indiscriminados y simultáneos por áreas funcionales y niveles jerárquicos, las cuales se vieron, hasta cierto punto, incluso peligrosas. En cambio se comprobó que otras tácticas fueron eficaces y por lo tanto positivas: 
* Comenzar con un esfuerzo consensuado entre todos: los sindicatos, directivos y representantes del nivel sobre el cual se va a determinar el "rightsize» a largo plazo, de forma que la empresa no pierda su «identidad corporativa».

- Proteger las competencias y áreas funcionales o de mercado más importantes y básicas para la organización.

* Poner énfasis en los equipos y grupos de trabajo, en la formación intensiva y en el liderazgo durante el tiempo que dure el proceso de reducción y después de él.

\section{Efectos derivados de la implantación del Downsizing}

Los estudios existentes acerca de los efectos derivados de la implantación del Downsizing son abundantes pero en muchas ocasiones contradictorios. Por ejemplo, Wyatt Associates encuestó a 1005 firmas que practicaron el Downsizing entre 1986 y 1991 encontrando que sólo el 46\% redujo costes, sólo el $32 \%$ ha incrementado actualmente beneficios, sólo el $22 \%$ incrementó la productividad y sólo el $17 \%$ redujo la burocracia (Bennett, 1991). Una explicación de lo anterior, podría ser que el Downsizing no ha sido dirigido de manera efectiva en la mayor parte de las firmas y, de este modo, no se han alcanzado las pretendidas reducciones de costes y el incremento en la eficiencia que se perseguían. Otra posible explicación sería que el Downsizing ha creado resentimiento y resistencia en las empresas, lo cual ha dificultado, más que favorecido, la competitividad en algunos países.

Contrariamente a los estudios anteriores, otros estudios recientes sobre Downsizing (entre los que se encuentra el de Byrne, 1994) han puesto de manifiesto que el $77 \%$ de aquellos que sobrevivieron al mismo sostenían que la reestructuración había tenido un impacto positivo sobre la productividad y sólo el $13 \%$ consideraba que había tenido un impacto adverso. Parecía claro. que la moral podía sufrir a corto plazo, pero la estrategia utilizada la mejoraba, especialmente cuando los despidos eran combinados con esfuerzos por romper con la burocracia. 
Otros beneficios resultantes citados por los encuestados fueron la aparición de un incremento en la calidad y servicio al cliente y una mayor disposición entre los supervivientes para aceptar riesgos.

Según este último autor, si se analizan detenidamente las consecuencias negativas asociadas generalmente al Downsizing por otros investigadores, se observa que los resultados están sesgados, no sólo por quienes han sido la muestra objeto del estudio sino también por el tamaño utilizado. Más aún, muchos de estos estudios están realizados inmediatamente después de proceder a los despidos, antes de que pudiera alcanzarse ningún resultado positivo.

En este apartado vamos a reseñar toda una serie de investigaciones que recogen los efectos más importantes y que, comúnmente, se desencadenan tras la implantación de un proceso de Downsizing.

\section{Efectos Organizacionales}

Algunas actividades de Downsizing suponen la reestructuración y eliminación de trabajos, encontrándonos entre otros con los siguientes fenómenos: utilización de funciones discontinuas, reducción de niveles jerárquicos, reingeniería de los procesos y fusión de unidades. Todo ello puede conducir a algún tipo de rediseño del trabajo tal como señalan los partidarios de la reingeniería de procesos, al argumentar que el resultado del Downsizing debería ser una organizción con menos personas que harían un trabajo más valioso.

Los estudios de Howard (1994) y Coleber (1994) señalan que con los procesos de Downsizing muchos economistas y directivos de niveles medios e intermedios estarán sobrando en las organizaciones, pues sus roles pasan a ser asumidos en gran parte por los niveles bajos. Un efecto de ello es la aparición de un modelo organizacional denominado "Organización horizontal" (McGoon, 1994) caracterizado por la supresión de niveles enteros de directivos de tipo medio, lo que ha supuesto un dramático cambio para los empleados con la consiguiente combinación de oportunidades y amenazas.

Para. McGoon aparece la necesidad de la "comunicación horizontal" como un fenómeno fundamental, y hasta ahora olvidado, de cara a asegurar la eficiente operación de casi todas las funciones organizacionales. En definitiva, todo esto está obliganco a un rediseño de estos puestos y a un replanteamiento de sus roles en la organización.

Según Hammonds (1994), una cuarta parte de todos aquellos empleados que trabajan actualmente realizan un trabajo temporal, a tiempo parcial, o con contratos mínimos. El «Outsourcing» es hoy día una estrategia más flexible que tener trabajadores o directivos empleados como fijos y/o a tiempo completo e implica un cambio en la cultura de las empresas. 
La estructura de las futuras corporaciones que surgirán tras todos los procesos actuales de Downsizing se caracterizará por su flexibilidad, la existencia de un cierto caos y la aparición de un entramado de equipos de trabajo unidos por potentes sistemas de información. Los individuos que formen parte de estas nuevas organizaciones tendrán que adaptarse a una forma de trabajar completamente nueva que incluirá en el acontecer diario procesos tales como: telecomunicaciones, existencia del trabajador *permanentemente temporal " y el "trabajo en casa” con una disponibilidad durante las veinticuatro horas del día (Jonhansen \& Swigart, 1994).

\section{Efectos sobre el personal que permanece en la empresa}

En 1992 la Right Associates encuestó a 1141 directivos del área de Recursos Humanos en Estados Unidos para determinar el efecto del Downsizing sobre «los empleados que permanecenn. La encuesta mostró que los empleados que permanecen en una organización en transición exhibian: falta de confianza en la misma, desconfianza hacia sus compañeros, altos niveles de stress y dudas sobre sus roles, (Pínola, 1994).

Así mismo, Moore (1994) y Navran (1994) sugieren en sus investigaciones que, tras un proceso de implantación de Downsizing, los empleados que quedan sucumben al desánimo y comparten un conjunto de reacciones comunes como son: resentimiento, escepticismo, pesimismo, desconfianza, vulnerabilidad, baja moral y baja motivación.

Algunas compañías han puesto en marcha programas para ayudar a los empleados que se quedan a "recomprometersew con los nuevos objetivos y con la nueva visión de la empresa (Véase Pínola 1994), y muchos son los autores que señalan a la "comunicación" como un factor clave para motivar al personal tras un proceso de Downsizing (véase por ej.: Stokes, 1994; McGoon, 1994; Schmenner, 1994, Flynn, 1994; Dreilinger, 1994, entre otros).

En un estudio realizado por Mone (1994), se examina cómo determinados factores a nivel individual, tales como la "autoconfianza», la *autoestiman, los "objetivos personales", usatisfacción por el puestow y ucompromiso para con la organización", afectan a las decisiones de los empleados relacionadas con la intención de abandonar una organización que atraviesa un proceso de Downsizing. Los resultados de la investigación indican que la «autoconfianza" y la "autoestima" ejercen un efecto positivo y directo en los intentos de los empleados por abandonar la organizacion, todo lo cual contrasta con la relación inversa encontrada entre estos autoconceptos (autoconfianza y autoestima) y el deseo de abandonar en organizaciones que no atraviesan estrategias de Downsizing. 
Los directivos de mayor nivel según Navran (1994), son los que deben explicar el por qué de los cambios producidos en la empresa y su necesidad vital de adoptarlos, para que así los empleados puedan entender mejor la situación y encarar los hechos con una actitud más positiva.

Según Hulsizer (1994), los ejecutivos deberían considerar el cambio como una oportunidad para hacer más eficiente la toma de decisiones, proporcionar más coordinación, y autorizar al personal para que sea más emprendedor. Sería adecuado formar un «equipo de transición" formado por los miembros más próximos y comprometidos con las nuevas ideas de los directivos, teniendo en cuenta que cada uno de ellos debería mostrarse receptivo y asumir que no tienen todas las respuestas, para así centrarse en la visión y valores necesarios para preparar una estrategia de transición.

En definitiva, la mayoría de los autores subrayan la idea de que los directivos han de involucrar a su personal en la tarea de reinventar la organización o su departamento y para ello nada mejor que establecer en primer lugar un buen sistema de comunicación interna, ya que el Downsizing crea en cierta medida un "caos comunicacional». Resaltan la importancia de una buena "comunicación" antes y durante el Downsizing, encaminada además a mejorar e intensificar las "relaciones laborales" con empleados y sindicatos.

Adoptando una visión más amplia, Cameron (1994) concluye de sus investigaciones que, para obtener éxito con el Downsizing, es necesario implantar y vigilar un eficaz sistema de gestión de los recursos humanos involucrando a los empleados y grupos de trabajo, promoviendo la comunicación e información compartida, el reconocimiento y las recompensas adecuadas, el entrenamiento y la formación, así como articulando una visión de empresa, y administrando el proceso de Downsizing en un clima de credibilidad y confianza. Todas estas medidas son vitales para el éxito de esta estrategia.

Los profesionales y directivos de Recursos Humanos juegan, según el autor, un papel central y clave a la hora de asegurar una buena implantación de eficaces estrategias de Downsizing, (Véase también Dreilinger, 1994).

\section{Efectos sobre el personal que abandona la empresa}

Por otra parte, es importante subrayar también los efectos causados por el Downsizing sobre los empleados que se ven abocados a abandonar la organización, así como sobre los que saben que en un futuro próximo han de hacerlo.

Las técnicas seguidas por algunas empresas consisten en encontrarles un puesto de trabajo a estos empleados, incluso acudiendo a empresas de «outplacement». Sin embargo, una buena salida consiste en crear sus propios negocios. 
Em este sentido es de destacar la interesante experiencia realizada por Ricardo Semler (1994) en una empresa brasileña denominada Semco. Esta empresa se enfrentaba a las dificultades derivadas de una economía en constante sobresalto y con altos niveles de inflación. Para superar la crisis economica la dirección se planteaba la necesidad de recortar los gastos de personal, pero en lugar de recurrir al método más expeditivo del despido, se emprendió un experimento novedoso y radical basado en la promoción de la creación de empresas satélites productoras de bienes y servicios que actuarían dentro de la empresa utilizando su equipo y valores y que venderían tanto. a Semco como a otras fábricas y cuyos miembros serían antiguos empleados, empleados a tiempo parcial y personas que no habían tenido ninguna conexión anterior con la empresa. Es importante señalar que este proceso difiere del conocido como Outsorcing, ya que es un sistema no contractual de asignación de tareas a corto plazo.

Este sistema puede parecer caótico y frustrante e incluso, en ocasiones, incontrolable. Requiere de actos diarios de fe y tiene amplias repercusiones en la cultura organizacional, con la desaparición, como primera consecuencia, de cualquier idea de seguridad.

Este nuevo modo de hacer se basa en una filosofía directiva que no cree en la jerarquía piramidal, que contempla la planificación estratégica como una barrera al éxito, que pone en entredicho la necesidad de control y que cuestiona el valor del crecimiento en cuanto éste no puede medirse por unos números que ignoran lo que el consumidor final piensa realmente del producto y lo que la gente que los produce piensa de la compañía.

Los tres nuevos valores de esta organización eran la participación de los empleados, la participación en beneficios y los sistemas de información.

Ahora la organización, en vez de ofrecer los contratos a extraños, contratará a sus propios ex-empleados. La empresa animaría a éstos a dejar la nómina de Semco y a establecer sus propias empresas satélites, actuando en un principio para Semco, alquilando su equipo y trabajando incluso en su misma planta. Las compensaciones adoptarían formas diversas tales como; pagos por contrato, royalties, comisiones, participación en beneficios etc... La empresa a su vez ofrecería ciertas garantías como la concesión de algunos contratos iniciales y el diferimiento de los alquileres del equipo y plantas en dos años.

Este programa tendría grandes ventajas para la empresa: reducción de los gastos de personal, disminución de los costes de inventarios que se distribuirían ahora entre los nuevos proveedores y pese a todo el mantenimiento de subcontratistas que conocen el negocio y la idiosincrasia de la compañía y de sus clientes. Además Semco se beneficiaría de la alta motivación empresarial de los empleados que estarían dispuestos a trabajar tardes y fines de 
semana cuando fuera necesario sin necesidad del requerimiento expreso por parte de los jefes.

Mientras que las ventajas para la organización eran claras no ocurría lo mismo con los empleados, ya que dependían en gran medida de los resultados de la economía pero, al ofrecérseles pocas alternativas factibles, muchos ex-empleados asumieron la propuesta.

A nuestro juicio, las dos ideas más interesantes subyacentes en este experimento son:

1. El valor negativo asignado a la estructura como generadora de una jerarquía que a su vez conlleva grandes limitaciones.

2. La fragmentación como una fuerza positiva para la organización. Frente a la existencia de un credo que recoge constantemente lo que la compañía es o quiere ser en un momento dado y el inmovilismo que esto origina. Semco apuesta por el dinamismo y la dispersión.

También resulta curioso el enfoque de Crandel (1994) respecto a los efectos causados por el Downsizing sobre aquellos empleados que saben que tienen que abandonar la empresa pero a los que se les pide que permanezcan el tiempo suficiente para garantizar la continuidad de la organizción después del Downsizing.

En la experiencia realizada sobre un grupo de trabajadores de este tipo durante un período de cuatro semanas, período al que llamó "Del pato cojo", se describireon cinco etapas: incredulidad, euforia, depresión, delirio y alivio. Este período era como un regalo consistente en cuatro semanas de paga y durante el cual se esperaba poco del que se iba, y si se esperaba daba vergüenza decirlo. Durante este tiempo el empleado adoptaba algunas formas de represalias, y al final, durante los últimos días, los afectados manifestaban que el trabajo había cambiado de modo radical y que, aunque no les gustaba que los echaran, tampoco querían quedarse.

Debido a las amplias repercusiones sociales, económicas, psicológicas y de toda indole que provocan los despidos han proliferado las investigaciones que se centran en el manejo más adecuado de éste y del desempleo en general, incluso de una forma previsional.

En este sentido y pese a las diferencias existentes en la metodología utilizada, en las muestras y en el diseño de las investigaciones, los científicos en el área de la "pérdida de empleo" (véase por ejemplo: Leana \& Feldman, 1992; Schweiges, Ivancevich \& Power, 1987), han identificado ocho políticas comunes que suelen usarse con buenos resultados y encaminadas todas ellas a yudar a los trabajadores para enfrentarse al desempleo de forma más realista y encontrar más rápidamente un nuevo empleo satisfactorio: 
1) Notificar el reajuste y consiguiente despido con antelación y tiempo suficientes. De esta forma los empleados que van a perder sus trabajos se pueden hacer a la idea y evitar las depresiones y ansiedades iniciales provocadas por la incertidumbre y la prisa. Esto les posibilita el poder contar con un plazo de tiempo suficiente durante el cual poder gestionar la búsqueda de un nuevo puesto de trabajo.

2) Asegurar el pago de los salarios y beneficios que correspondan. Así los empleados desplazados o despedidos tendrán una seguridad económica que les cubre de posibles pérdidas financieras o estados de necesidad.

3) Crear programas de educación y reentrenamiento. Para que los empleados despedidos puedan desarrollar nuevas habilidades y destrezas que les ayuden en nuevos trabajos o puesto en diferentes empresas y/o diferentes sectores de actividad.

4) Proporcionar asistencia de Outplacement. Así estos empleados pueden aprender (y/o se les puede asesorar) cómo localizar nuevas oportunidades de trabajo y nuevos mercados proveedores de empleo.

5) Anunciar las decisiones de despido de manera clara, directa y con empatía. Si se hace de esta forma, se evitan malos entendidos y se posibilita que los empleados conozcan claramente las razones, puedan conservar su dignidad y puedan recibir apoyo social de sus compañeros y de aquellos empleados que sobreviven o permanecen en la organización.

6) Prestar atención a los supervivientes del despido. Para que aquellos empleados que permanecen en la organización continúen con un buen nivel de compromiso y productividad.

7) Practicar una planificación de los recursos humanos que considere alternativas al despido permanente o de gran escala. De esta forma se minimizará el número de empleados despedidos o desplazados en el futuro.

8) Proponer la colaboración entre las organizaciones del sector privado y del sector público para proporcionar servicios a los empleados despedidos. Se pretende así que éstos puedan recibir la mayor asistencia y ayuda posibles con la mayor prontitud. 
En un trabajo posterior, realizado en el año 1994. Feldman \& Leana llevaron a cabo una investigación más completa sobre las principales (o mejores) prácticas en la gestión de los despidos y que de forma resumida se detallan a continuación:

1. Implantar sistemas previsionales para identificar condiciones y señales de despidos potenciales.

2. Contar con sistemas de planificación de recursos humanos que se centren más en el desarrollo y perfeccionamiento de la fuerza de trabajo que en un despido permanente.

3. Establecer una comunicación honesta, directa y empática sobre el despido con los empleados.

4. Identificar aquellos empleados que podrán beneficiarse con programas de asistencia y hacerles más fácil el despido y la prestación de servicios de apoyo.

5. Llevar a cabo una identificación temprana de aquellas plantas, departamentos o secciones de la empresa con problemas concretos de despidos y diseñar diversos planes de intervención dirigidos a resolver problemas específicos en cada lugar.

6. Involucrar a los empleados (a despedir) en el diseño y la implantación de los programas de asistencia y ayuda.

7. Los programas de apoyo social, no deben ser diseñados sólo para disminuir el estrés psicológico sino que deben centrarse también, en generar y conseguir un compromiso por parte de estos empleados en la búsqueda de un nuevo trabajo.

8. Establecer con tiempo suficiente (anticipadamente), programas de asistencia financiera para despedidos, tales como planes de pensiones, reparto de beneficios e indemnizaciones, así como una adecuada información sobre indemnizaciones públicas (del gobierno) en casos de despidos.

9. Implantar programas de "outplacement» que incluyan tanto la evaluación de las habilidades de las personas, como determinados programas de formación en técnicas de búsqueda de empleo y servicios de apoyo a la misma.

10. Establecer programas de formación que centren la atención de los empleados y les sensibilicen en la necesidad o conveniencia de cambiar sus carreras y perfiles profesionales. 
11. Establecer programas de asistencia a los empleados que permanecen en la empresa, al objeto de reconstruir la moral y el compromiso psicológico para con la misma, así como dirigidos a un incremento de sus niveles de productividad.

12. Arbitrar una ayuda financiera por parte de la empresa a aquellas plantas, departamentos o secciones afectadas por el Downsizing.

13. Contemplar en la estrategia de Downsizing, el grado de responsabilidad social que ha de asumir la empresa con las comunidades locales afectadas, diseñando acciones concretas que minimicen el impacto adverso o negativo del despido en dichas comunidades.

14. Diseñar un programa de asistencia a los despedidos en coordinación y colaboración con las agencias gubernamentales, sindicatos, grupos de acción local e instituciones educativas locales.

\section{Conclusiones}

Hemos visto a lo largo de este trabajo como el Downsizing puede ser una estrategia adecuada en el entorno actual en que se desenvuelven las organizaciones. No obstante, los efectos no deseados que aparecen tras su implantación pueden echar por tierra todos los intentos de mejora de la eficiencia, productividad y/o competitividad que le dieron origen.

El Downsizing provoca un "terremoto» en la organización, siendo el pilar formado por el personal uno de los más afectados. Una fuerza laboral insegura, desmotivada, ignorante de su nuevo papel y preguntándose ¿cuándo me tocará a mí? no puede conseguir los altos objetivos perseguidos.

Los directivos de Recursos Humanos en particular, pero todos en general, tienen que asumir un papel activo $y$, a través de la "comunicación de doble sentido" como elemento clave, deben involucrar al personal en el proceso de Downsizing: articulando una nueva visión de la organización, aclarando el papel de cada individuo en la empresa y promoviendo un ambiente de credibilidad y confianza. Sólo de esta forma el Downsinzing podrá constituirse en una alternativa válida para salvar a la empresa de situaciones dificiles permitiéndola recuperar una posición fuerte con unos procesos productivos rediseñados y con una fuerza laboral activa, motivada e integrada con la nueva imagen, estructura y quehacer de la organización.

El trabajo tal como es conocido hoy no formará parte de la realidad económica del mañana. Los trabajadores tendrán que encontrar nuevas formas de trabajar, ya que la figura tradicional del empleado leal no tendrá ninguna. 
vigencia. Estos tendrán que aprender a vivir con altos grados de incertidumbre y deberán sustituir la seguridad externa perdida, por una nueva seguridad que proceda de ellos mismos, considerándose así mismo como negocios que deben planificar y gestionar.

El núcleo del nuevo contrato social será la independencia y la interdependencia, frente a la clásica dependencia. En consecuencia, el nuevo credo propuesto para enfrentarse al nuevo entorno propone el crecimiento personal, el desarrollo de habilidades, el aprendizaje continuo y la colaboración, ya que los individuos no trabajarán "para una organización" sino que se "unirán" a ella para desarrollar una relación basada en la igualdad y el interés recíproco.

\section{Bibliografía}

ANONYMUS: Salaried workers are targets of corporate downsizing. HR Focus. Vol. 71. Jun. 1994. Pág. 21.

BEHN, R.: How to terminate publicy: a dozen hints for the would-be terminator. En Levine, C. (Ed.). Managing fiscal stress. 1980. Págs. 310-322. Chatham. N.J: Chatham House.

BENNET, A.: Downsizing doesn't necessarily bring an upswing in corporate profitability. Wall Street Journal. Jun. 1991. pág.B1.

BLAU, P.M.: A formal theory of differentiation in organizations. American Sociological Review. 1970. Vol. 35 págs. 210-218.

BROCKNER, J.: The effects of work layoff on survivors: research, theory, and practice. En Staw, B,; y Cummings, L. (Eds), Research on organizational behavior. Vol. 10, 1988. Págs.00-00. Greenwich. CT: JAI Press.

BuHLer, P.: Downsizing. Supervision. Vol. 55 Nov. 1994. págs. 24-26.

BYRNE, J.: There is an upside to downsizing. Business Week. Mayo 9, 1994, pág. 69. CAMERON, K.: Strategies for successful organizational downsizing. Human Resource Management. Vol. 33. Verano 1994, págs. 189-211.

Cameron, K.; Freeman, S.; Mishra, A.: Downsizing and redesigning organizations. En: HUBER, G.: GLICK, W.: Organizational change and redesigs. Oxford University Press. N. York. 1993. págs.: 19-63.

COLEBERD, R.: The business economist at work: The economist as entrepreneur. Business Economics. Vol. 29. Oct. 1994. págs. 54-57.

CRANDELl, S.: Job purgatory. Working Woman, Vol. 19. Junio 1994, págs.: 44-47. DREIINGER, C.: "Fear and loathing after downsizing... What can managers do? Tapping the Network Journal. Vol. 5 Verano 1994. págs.: 8-10.

FELDMAN, D.C. \& LEANA, C.R.: Better paractices in managing layoffs. Human Resource Management. Vol. $33 \mathrm{n}^{\circ} 2,1994$, págs. 239-260.

FLYNN, G.: Downsizing survivors can get back on track with help. Personnel journal.

Vol. 73. Noviembre 1994, pág.: 18. 
GLMORE, T. y HIRSCHORN, I.: Management challenges under conditions of retrenchment. Human Resource Management. Vol. 22, 1983, págs.: 341-357.

GREINER, L.: Evolution and revolution as organizations grow. Harvard Business Review. Vol. 49, 1972, págs.: 37-46.

HAMmoNDS, K.: The new world of work. Bussines Week. $\mathrm{n}^{\circ} 3394.17$ octubre 1994. págs.: 76-87.

HIRSCHORN, L. y Asociados: Cutting back: retrechment and redevelopment in human and commnity services. San Francisco: Jossey-Bass, 1983.

HITT, M.; KEATS, B.; HARBACK, H.; NIXON, R.: Rightsizing: Building and maintaining strategic leadership and long-term compettiveness. Organizational Dynamics. Vol. 23. Otoño 1994. Págs. 18-32.

HowARD, L.: Downsizing spawns risk, expert says. National Underwriter. Vol. 98. Oct. 17. 1994. págs.: 7-16.

Hulsizer, G.: Successful transitions. Executive Excellence. Vol. 11. Abril 1994. págs.: 17-18.

JOHANSEN, R. \& SWIGART, R.: Upsizing the individual in the Downsized Organization. Reading Massachusetts: Addison-Wesley, 1994.

KRANTZ, J.: Group processes under conditions of organizational decline. Journal of Applied Behavioral Science. Vol. 21. 1985. págs. 1-17.

LEANA, C.R. \& FELDMAN, D.C.: Coping with job loss: How individuals, organizations, and comunities respond to layoffs. New York. 1992. Macmillan/Lexington Books.

MARgulIS, S.T.: Bad news, good news about downsizing. Managing Office Technology. Vol. 39. Abril 1994, págs.: 23-24.

MCCune, J.T.; BEATTY, R.W. y Montagno, R.V.: Downsizing: practices in manufacturing firms. Human Resource Management Journal. Vol. 27, 1988. págs.: 145-161.

McGoon, C.: After downsizing... then what? Communication World. Vol. 11. Mayo 1994, págs.: 16-19.

-, What role shall we play today? Communication World. Vol. 11. Agosto 1994. págs.: 12-16.

MCKINLEY, W.: Complexity and administrative intensity: the case of declining organizations. Administrative Science Quarterly. Vol. 32. 1987, págs.: 87-105.

MILES, R.H. y CAMERON, K.S.: Coffin nails and corporate strategies. Englewood Cliffs, NJ: Prentice-Hall. 1982.

MONE, M.: Relationships between self-concepts, aspirations, emotional responses, and intent to leave a downsizing organization. Human Resource Management. Vol. 33. Verano 1994. págs.: 281-298.

MOORE, T.: Rightsizing: living with the new reality. Healthacare Financial Mangement. Vol. 48. Sep. 1994, págs. 48-54.

Moravec, M.: The right way to rightsize. Industry Week. Vol. 243. Sep. 5. 1994. Pág.: 46.

NaVran, F.: Surviving a downsizing. Executive Excellence. Vol. 11. Jul. 1994. págs: 12-13.

PINOLA, R.J.: Building a winning team after a downsizing. Compensation and Benefits! Management. Vol. 10. Invierno, 1994. págs.: 54-59. 
QuinN, R.E. y CAMERon, K.: Organizational life cycles and shifting criteria of effectiveness. Management Science. Vol. 29. 1983. págs.: 33-51.

SCHMENNER, R.; LACKEY, Ch.: Slash and burn 'doesn't kill weeds: Other ways to downsizw the manufacturing organization. Business Horizons. Vol. 37. Jul./Ag. 1994. págs.: 80-87.

SchWeIger, D.M.; IVANCEVICH, J.M. \& Power, F.R.: Executive actions for managing human resources before and fater acquisitions. Academy of Management Executive. 1. 1987, págs.: 127-138.

SEMLER, R.: Why my former employees still work for me. Harvard Business Review. Enero-Febrero, 1994. págs.: 64-74.

STOKES, S.: Life after rightsizing. Information Systems Management. Vol. 11. Otoño 1994, págs.: 69-71.

WeITZEl, W y JoHNSON, E.: Decline in organizations: a literature integration and extensión. Administrative Science Quarterly. Vol. 34. 1989. págs.: 91-109. 